\title{
China's Forest Coverage Rate Forecasting Model Based on Gray System Theory
}

\author{
Zhuoshi $\mathrm{Li}^{1, \mathrm{a}}$, Wenqian Wang ${ }^{2, \mathrm{~b} *}$, Lizong Cao ${ }^{3, \mathrm{c}}$ and Zhengwei Liu ${ }^{4, \mathrm{~d}}$ \\ 1,2,3,4 Jilin Agricultural University, Changchun, Jilin, 130118, China

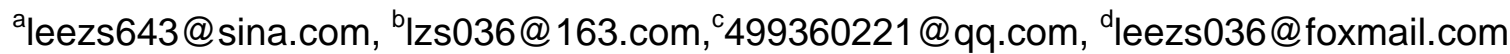

Keywords: the forest coverage rate;grey system theory; $\operatorname{GM}(1,1)$ model; residual correction method

Abstract. In this paper, according to China in 1973-2013 in eight forest inventory published forest cover of statistical data. Using gray system theory GM $(1,1)$ prediction model for China's forest coverage rate forecast. Then, the use of residues correction method for the prediction model is optimized to ensure the accuracy of the model by inspection. The results show a gray optimized forecasting model is applicable to China's forest coverage rate of medium and long term forecasting and prediction with high accuracy, and make the necessary preparations for the subsequent research forest coverage.

\section{Introduction}

Forest resources are the material basis of ecological the Construction and are the fundamental to sustainable development of forestry. Strengthen the protection of forest resources to build a harmonious society is of great significance, and the forest coverage rate is reflected in the abundance of forest resources and ecological balance status an important indicator. At the same time, according to the eighth national forest inventory results of Chinese Forestry Administration released in 2014, is currently China's forest coverage rate of $21.63 \%$, this data is far below the average of $31 \%$ of the world, per capita forest area is only a quarter of the world[1][5]. Therefore, forest coverage forecasting on China's economy to improve people's living standards. The scientific development of forest management plans and establish a technology support system, and continuously improve multifaceted significance of forest cover, rich in forest resources will seek the best forest coverage[4].

In order to get effective forest coverage prediction system, an objective of the development trend of China's forest coverage rate forecast. We use the theory and statistical methods and some basis of predictions, forecasting methods reviewed research on forest coverage domestic and foreign documents, Using the existing data, GM $(1,1)$ model to predict China's forest coverage by scientific gray system, and optimize the use of with higher accuracy predictive model.

\section{The Basic Theory of Grey System [2]}

Definition 1 Let $X^{(0)}=\left\{x^{(0)}(1), x^{(0)}(2), \ldots, x^{(0)}(n)\right\}, X^{(1)}=\left\{x^{(1)}(1), x^{(1)}(2), \ldots, x^{(1)}(n)\right\}$, $x^{(0)}(k)+a x^{(1)}(k)=b$

Definition 2 Let $X^{(0)} \quad X^{(1)}$ such as definition, as shown in the (1),

$$
\begin{aligned}
& Z^{(1)}=\left\{z^{(1)}(2), Z^{(1)}(3), \ldots, Z^{(1)}(n)\right\}, z^{(1)}(k)=\frac{1}{2}\left(x^{(1)}(k)+x^{(1)}(k-1)\right), \quad k=2,3, \ldots, n \\
& x^{(0)}(k)+a z^{(1)}(k)=b
\end{aligned}
$$

For the basic form of the $\operatorname{GM}(1,1)$ model.The parameter $(-a)$ is the development coefficient, $b$ is grey action coefficient .

Theorem 1 Set a negative sequence, $X^{(0)}=\left\{x^{(0)}(1), x^{(0)}(2), \ldots, X^{(0)}(n)\right\}$,Among them $x^{(0)}(k) \geq 0, k=1,2, \ldots, n ; X^{(1)}$ is $X^{(0)} 1-A G O ; x^{(0)}(k) \geq 0, k=1,2, \ldots, n ; X^{(1)}$ is $X^{(0)} 1-A G O$ for the sequence $X^{(1)}=\left\{x^{(1)}(1), x^{(1)}(2), \ldots, X^{(1)}(n)\right\}$, 
Among them $x^{(1)}(k)=n \sum_{i=1}^{k} x^{(0)}(i), k=1,2, \ldots, n \quad ; \quad X^{(1)}$ As close to the mean of generating , $Z^{(1)}=\left\{z^{(1)}(2), z^{(1)}(3), \ldots, z^{(1)}(n)\right\}$,

$$
\begin{aligned}
& z^{(1)}(k)=\frac{1}{2}\left(x^{(1)}(k)+x^{(1)}(k-1)\right), k=2,3, \ldots, n \\
& \text { If } \quad \hat{a}=(a, b)^{T}, \\
& Y=\left[\begin{array}{c}
x^{(0)}(2) \\
x^{(0)}(3) \\
\vdots \\
x^{(0)}(n)
\end{array}\right], B=\left[\begin{array}{cc}
-z^{(1)}(2) & 1 \\
-z^{(1)}(3) & 1 \\
\vdots & \vdots \\
-z^{(1)}(n) & 1
\end{array}\right]
\end{aligned}
$$

The GM(1,1) model of least squares estimate parameters,

$$
x^{(0)}(k)+a x^{(1)}(k)=b, \hat{a}=\left(B^{T} B\right)^{-1} B^{T} Y
$$

Definition 3 Let $X^{(0)}$ to a negativesequence, $X^{(1)}$ for the sequence of $X^{(0)}$ generate $X^{(1)} 1-A G O$ sequence, $Z^{(1)}$ for close to the mean of,

$$
(a, b)^{T}=\left(B^{T} B\right)^{-1} B^{T} Y, \frac{d x^{(1)}}{d t}+a x^{(1)}=b
$$

$X^{(1)}$ for the sequence of $X^{(0)}$ generate $X^{(1)} 1-A G O$ sequence, $Z^{(1)}$ for close to the mean, Says $(a, b)^{T}=\left(B^{T} B\right)^{-1} B^{T} Y$ As the model equation of bleaching be also called shadow equation .

Theorem 2 Let $B, Y, \hat{a}$ as described above theorem $1, \hat{a}=(a, b)^{T}=\left(B^{T} B\right)^{-1} B^{T} Y$,

1) An albinoequation solution into time response function is also An albino equation solution into the time response function is braking.

2) $\frac{d x^{(1)}}{d t}+a x^{(1)}=b$

3) Model $x^{(0)}(k)+a z^{(1)}(k)=b$ of time sequence for accordingly

$$
\hat{x}^{(1)}(k+1)=\left(x^{(0)}(1)-\frac{b}{a}\right) e^{-a k}+\frac{b}{a} \quad k=1,2, \ldots, n
$$

4) Reduced value

$$
\hat{x}^{(0)}(k+1)=x^{(1)}(k+1)-\hat{x}^{(1)}(k)=\left(1-e^{a}\right)\left(x^{(0)}(1)-\frac{b}{a}\right) e^{-a k}, k=1,2, \ldots, n
$$

\section{Forest Coverage Rate GM (1,1) Prediction Model}

In this paper, the establishment of the model chosen for the data in 1973-2013 released eight Forest Resource Inventory Data .

From the Forest Resource Inventory Data We can get the original data sequence[3].

$x^{(0)}(k)=(12.10,12.00,12.98,13.92,16.55,18.21,20.63,21.63)$

When the sequence of data start and end point is null. It can not fill the vacancies generated using the mean, instead of considering some other methods.It is a very common method of the pole ratio generating fill sequence approach.Solving $\lambda(k)$. $\lambda(k)=\frac{x^{(0)}(k-1)}{x^{(0)}(k)}=(1.0083333,0.9244992,0.9324713,0.8470876,0.9088413,0.8826951,0.9537679)$

Obtained from the feasible interval $(0.846817,1.1813604)$, the $\lambda(k)$ are within feasible interval. The Accumulated Generating Operation sequence $1-A G O$. 


$$
\begin{aligned}
& x^{(1)}(k)=x^{(1)}(k-1)+x^{(0)}(k) \\
& =\left(x^{(1)}(1), x^{(1)}(1), x^{(1)}(2), x^{(1)}(3), x^{(1)}(4), x^{(1)}(5), x^{(1)}(6), x^{(1)}(7), x^{(1)}(8)\right) \\
& =(12.10,24.10,37.08,51.00,67.55,85.76,106.39,128.02) \\
& B=\left[\begin{array}{cc}
-0.5\left(x^{(1)}(1)+x^{(1)}(2)\right) & 1 \\
-0.5\left(x^{(1)}(2)+x^{(1)}(3)\right) & 1 \\
\ldots & \ldots \\
-0.5\left(x^{(1)}(7)+x^{(1)}(8)\right) & 1
\end{array}\right]=\left[\begin{array}{cc}
-18.100 & 1 \\
-30.590 & 1 \\
\ldots & \ldots \\
-117.205 & 1
\end{array}\right] \\
& Y=\left[x^{(0)}(2), x^{(0)}(3) . \cdots x^{(0)}(8)\right]^{T}=(12.00,12.98,13.92,16.55,18.21,20.63,21.63)^{T}
\end{aligned}
$$

$-a=0.216319608$ is development coefficient, $b=11.37626162$ is grey action coefficient.

According to the theory and formulas,we can get temporal response function.

$$
\hat{x}^{(1)}(k+1)=\left(x^{(0)}(1)-\frac{b}{a}\right) e^{-a k}+\frac{b}{a}=64.6901 \cdot e^{0.2163 k}+52.5901
$$

Get predicted values of the original sequence.

$\hat{x}^{(0)}(k)=(12.10,12.68,13.01,13.78,14.56,15.62,19.39,21.06,24.08,28.18)$.

The forecast data and the error value as shown in Table 1.

Table 1 China's Forest Coverage Rate Prediction Table

\begin{tabular}{|c|c|c|c|c|}
\hline Year & $\begin{array}{c}\text { Prediction Forest } \\
\text { Coverage Rate }\end{array}$ & $\begin{array}{c}\text { The original Coverage } \\
\text { Rate }\end{array}$ & The Absolute Error & $\begin{array}{c}\text { The Relative Error } \\
(\%)\end{array}$ \\
\hline $1973-1976$ & 12.10 & 12.10 & 0 & 0 \\
\hline $1977-1981$ & 12.68 & 12.00 & 0.68 & 5.667 \\
\hline $1984-1988$ & 13.01 & 12.98 & 0.03 & 0.231 \\
\hline $1989-1993$ & 13.78 & 13.92 & 0.14 & 1.006 \\
\hline $1994-1998$ & 14.56 & 16.55 & 1.99 & 12.024 \\
\hline $1999-2003$ & 15.62 & 18.21 & 2.59 & 14.223 \\
\hline $2004-2008$ & 19.39 & 20.63 & 1.24 & 6.011 \\
\hline $2009-2013$ & 21.06 & 21.63 & 0.57 & 2.635 \\
\hline $2014-2018$ & 24.08 & $/$ & $/$ & $/$ \\
\hline $2019-2023$ & 28.18 & $/$ & $/$ & $/$ \\
\hline
\end{tabular}

\section{Residual Correction Method of the Optimization Processing Model}

In order to obtain with higher accuracy, we can consider the establishment the model of the traditional model residuals amending.

Get the residual sequence $e=(-0.68,-0.03,0.14,1.99,2.59,1.24,0.57)$.

$\hat{\lambda}(k)=\frac{x^{(0)}(k-1)}{x^{(0)}(k)}=(0.0441178,4.666667,14.214286,1.3015076,0.4787645,044516129)$

From the feasible interval $(0.846817,1.1813604)$, e sequence can not judged feasibility. Taken the appropriate offset value $Q$ satisfing the judge. $Q=200$,get the translation sequence.

$\Gamma=(199.32,199.97,200.14,201.99,202.59,201.24,200.57)$

$-\hat{a}=0.6182186, \hat{b}=21.6894786$

$\hat{\mathrm{e}}^{(1)}(k+1)=\left(\mathrm{e}^{(0)}(1)-\frac{\hat{b}}{\hat{a}}\right) e^{-\hat{a} k}+\frac{\hat{b}}{\hat{a}}=163.1386 \cdot e^{0.6182186 k}+36.1814$

Corrected model. $\hat{x}^{(1)}(k+1)=\left(\mathrm{x}^{(0)}(1)-\frac{b}{a}\right) \cdot e^{-a k}+\frac{b}{a}+\delta(k-1) \cdot(-\hat{a}) \cdot\left(\mathrm{e}^{(0)}(1)-\frac{\hat{b}}{\hat{a}}\right) \cdot e^{-\hat{a}(k-1)}$

The Gray predicted value after compensation. 
Table 2 The Predicted Residual Error Correction Method and Relative Error Table

\begin{tabular}{|c|c|c|c|}
\hline Year & After Compensation Prediction Forest Coverage Rate & The Absolute Error & The Relative Error (\%) \\
\hline $1977-1981$ & 12.00 & 0 & 0 \\
\hline $1984-1988$ & 12.68 & 0.3 & 2.311 \\
\hline $1989-1993$ & 14.02 & 0.1 & 0.718 \\
\hline $1994-1998$ & 15.58 & 0.97 & 5.861 \\
\hline $1999-2003$ & 16.23 & 1.98 & 10.873 \\
\hline $2004-2008$ & 18.77 & 1.869 & 9.016 \\
\hline $2009-2013$ & 21.12 & 0.51 & 2.358 \\
\hline
\end{tabular}

From the above table shows the average error optimization using the residual gray forecasting model to predict Forest coverage rate for the fitting accuracy.

\section{Acknowledgement}

Jilin Province Innovation and Entrepreneurship Students Training Program Project.

Jilin Agricultural University Undergraduate Science and Technology Innovation Fund Project.

\section{References}

[1] Worrell.R. and Malcolm.D.C Productivity of sake spruce in Northern Britain.The effects of elevation and climate.(1990) 56-58 .

[2] Xie.N.M,The grey system model research. Nanjing university of aeronautics and astronautics. (2008)9-17.

[3] Bao.Y.K,Li.N,Matlab Mathematical Statistics and Data Processing,Northeastern University Press,Beijing,2008.

[4] Song,Y.C,The problems existing in the real estate industry in our country and the development countermeasures.Gansu Agricultura.18(2010)37-40.

[5] Yang.Z.H,Applied Mathematical Statistics,first ed.,Beijing University of Technology Press. Beijing, 2005. 